Ciência Florestal, Santa Maria, v. 7, n. 1, p. 101-126, 1997

ISSN 0103-9954

\title{
AVALIAÇÃO DA PRODUÇÃO EM VOLUME TOTAL E SORTIMENTO EM POVOAMENTOS DE Pinus taeda L. SUBMETIDOS A DIFERENTES CONDIÇÕES DE ESPAÇAMENTO INICIAL E SÍTIO
}

\section{YIELD AND ASSORTMENT EVALUATION OF Pinus taeda $\mathrm{L}$. STANDS WITH DIFFERENT INITIALS SPACING AND SITE- QUALITY}

\author{
Fernando dos Santos Gomes ${ }^{1}$ Romualdo Maestre ${ }^{2}$ \\ Carlos Roberto Sanquetta ${ }^{3}$
}

\section{RESUMO}

É apresentada a avaliação do volume total e volume por sortimento por unidade de área, em povoamentos de Pinus taeda L. submetidos a condições distintas de sítio e de densidade de plantio. Os dados são provenientes de um experimento de espaçamento inicial em Pinus taeda L., instalado no município de Jaguariaíva - PR, na Fazenda Lageado, de propriedade da empresa "Pisa Florestal". Os espaçamentos iniciais utilizados foram: tratamento $1(2,5 \mathrm{x}$ $1,2 \mathrm{~m})$, tratamento $2(2,5 \times 2,0 \mathrm{~m})$, tratamento $3(2,5 \times 2,8 \mathrm{~m})$, tratamento 4 $(2,5 \times 3,6 \mathrm{~m})$ e tratamento $5(2,5 \times 4,4 \mathrm{~m})$. Seis blocos foram relacionados com as condições de sítio. A produção foi avaliada através do Delineamento em Blocos Casualizados para a idade atual dos povoamentos, que corresponde a 8,75 anos. Resultados de simulações da produção de cada tratamento em condições extremas de sítio foram obtidos através de um simulador de crescimento e produção denominado SISPINUS. As simulações de produção foram feitas até a idade de 15 anos, desconsiderando-se práticas de desbastes. $\mathrm{Na}$ idade atual dos povoamentos, a análise do sortimento demonstrou que espaçamentos mais amplos implicam em um aumento do volume disponível para fins mais nobres, como serraria e laminação. As simulações indicaram que, em idades futuras, os tratamentos exercem efeitos similares na produção de madeira grossa, devido à não realização de desbastes. Espaçamentos

1 Engenheiro Florestal, Acadêmico de Mestrado em Manejo Florestal do Curso de Pós-Graduação em Engenharia Florestal. UFPR. 80.035-010. Curitiba. PR.

2 Engenheiro Florestal, M.Sc. Gerente Técnico da PISA Florestal S.A.

3 Engenheiro Florestal, M.Sc., Ph.D., Professor do Departamento de Silvicultura e Manejo. UFPR. 80.035-010. Curitiba. PR. 
mais densos aumentaram a produção em volume total, em qualquer idade de avaliação. A análise do delineamento na idade atual dos povoamentos indicou claramente o efeito dos blocos, tanto na produção em volume total como no volume para serraria. As simulações realizadas permitiram concluir que o efeito do sítio é mais acentuado na produção de madeira de maior valor agregado.

Palavras-chave: espaçamento inicial, sítio, Pinus taeda, simulação.

\begin{abstract}
This study presents an evaluation of total and assortment volumes per area unit, in stands of tinus taeda L. under different site conditions and planting density. The data are from a initial pacing experiment of Pinus taeda L. at Fazenda Lageado, Jaguariaiva-PR, owned by Pisa Florestal ompany. The initial spacings used were: treatment $1(2,5 \times 1,2 \mathrm{~m})$; treatment $2(2,5 \times 2,0 \mathrm{~m})$; treatment $3(2,5 \times 2,8 \mathrm{~m})$; treatment $4(2,5 \times 3,6 \mathrm{~m})$; and treatment $5(2,5 \times$ $4,4 \mathrm{~m})$. Six blocks were related to site conditions. The volume production was evaluated through the Randomized Complete Block Design to the present age of the stands, that is 8,75 years. Simulation results of each treatment production under extreme conditions of site were got by a growing and production imulator called "SISPINUS". The production simulations were made until 15 years old, not considering thinnings. At the present age of the stands, assortment analysis showed that large spacing implies an increasing sawmill and veneer volumes. The simulation showed that, in the future, the treatments would have a similar effect on thick wood production, if no thinning was carried out. Narrower spacing increased total volume production, at any age of evaluation. The design analysis at the present age clearly showed the effect of the blocks on total volume production as well as the volume for sawmill wood production. The simulations allowed to conclude that the site effect is more conspicuous in higher economically valuable wood production. Key words: initial spacing, site, Pinus taeda, simulation.
\end{abstract}

\title{
INTRODUÇÃO
}

A extensa área plantada com espécies do gênero Pinus no Brasil, o múltiplo uso desses povoamentos florestais e a divergência com relação ao manejo mais adequado à finalidade da madeira proveniente de desbastes 
e corte raso, são alguns dos aspectos que exigem a necessidade de estudos de regimes silviculturais adequados a cada situação. No planejamento da produção para qualquer finalidade industrial, considerando ou não práticas de poda e desbaste, a definição do regime de manejo exige invariavelmente a escolha adequada da densidade de plantio.

A definição do espaçamento inicial depende da destinação que será dada à madeira proveniente do corte. Se o objetivo é produzir madeira para fins mais nobres, como laminação e serraria, a utilização de um espaçamento inicial mais amplo toma-se vantajosa. No entanto, a condução de povoamentos para produção de biomassa ou de madeira destinada exclusivamente ao processo para fábrica de papel, requer uma maior densidade inicial (espaçamentos menores) para maximizar a produção. Regimes de desbaste e/ou poda a serem efetuados no povoamento devem ser levados em consideração na definição do espaçamento inicial.

Quando o propósito da produção de madeira de um talhão for o processamento químico ou mecânico de cavacos / fibras para a produção de celulose e papel, chapas de partículas de madeira glomerada, etc., deve-se reduzir o número de desbastes ao mínimo, podendo se evitar mesmo, por completo, se possível, a adoção de qualquer regime de desbaste (AHRENS, 1985). O autor sugere uma rotação de 15 anos para Pinus em regime direto, sem desbaste.

A qualidade do sítio florestal também deve ser considerada na definição de regimes de manejo. Conforme HUSCH et al., (1982), a qualidade do sítio expressa a produtividade média que uma determinada área apresenta no crescimento de árvores. Uma forma comum de expressar a qualidade de sítio de forma relativa é dividir a área em três a cinco classes de sítio. As características de cada classe devem ser definidas para possibilitar a classificação de uma área florestal.

DAVIS (1966) cita uma definição de sítio segundo a "Society of American Foresters" (1958): "os fatores ecológicos de uma área, com relação à capacidade de produzir florestas ou outra vegetação; ou a combinação de condições bióticas, climáticas e de solo de uma área." A qualidade do sítio refere-se, desta forma, à combinação de todos os fatores biológicos e ambientais que afetam o crescimento das árvores.

$\mathrm{O}$ conhecimento das classes de produtividade existentes em uma área florestal torna-se imprescindível, pois possibilita considerar no planejamento a combinação dos efeitos que o sítio e o regime de manejo 
exercem sobre a produção. Um espaçamento inicial ou mesmo um regime de manejo pode ser concebido para cada classe de produtividade.

A definição de práticas de manejo em povoamentos florestais já implantados, terá fundamentos técnicos mais apropriados quando houver meios de simular a produção futura.

Segundo OLIVEIRA (1995), é fundamental que um modelo de prognose do crescimento e da produção de povoamentos de Pinus possibilite a simulação de desbastes e a separação das estimativas de volume total de madeira em volumes parciais, estimados especificamente para segmentos dos troncos com dimensões adequadas a cada finalidade industrial. Para que isto seja possível, este modelo deve estar baseado em funções de distribuição de probabilidades que descrevem as distribuições de diâmetro e altura das árvores do povoamento em diversas condições de sítio, idade e número de árvores por hectare. O autor desenvolveu o simulador SISPINUS - Versão 2.0, elaborado a partir da primeira versão do "NCSU Simulador-SISPINUS", para possibilitar, além da simulação de desbastes e do crescimento e produção anual do povoamento, o sortimento de madeira por classe diamétrica.

As ferramentas existentes para simulação de crescimento e produção podem ser utilizadas no planejamento florestal, para verificação da produção esperada em idades futuras, em diferentes condições de sítio e densidade. Para o caso particular do presente estudo, a desconsideração de práticas de desbaste e a realização do corte raso aos 15 anos de idade restringem substancialmente o número de simulações, que deverá contemplar apenas as combinações de espaçamento inicial e sítio.

Os objetivos do presente trabalho podem ser resumidos como segue:

a) Quantificar o volume total e o volume para serraria por hectare em um experimento de espaçamento inicial de Pinus taeda, na idade de 8,75 anos, visando detectar diferenças de produção na utilização de diferentes densidades de plantio para esta idade;

b) Avaliar o efeito do índice de sítio sobre a produção em volume total e volume para serraria, na idade de 8,75 anos;

c) Identificar os efeitos exercidos pelos tratamentos de espaçamento inicial e por condições extremas de sítio na produção volumétrica e no sortimento em idades futuras, através de simulações realizadas com o programa SISPINUS. 


\section{MATERIAL E MÉTODOS}

\section{Descrição do experimento}

O experimento foi instalado no município de Jaguariaíva - PR, na Fazenda - Lageado, de propriedade da "Pisa Florestal", Projeto Pisa 26, talhão 11. A implantação ocorreu entre os dias 28 de outubro e 09 de novembro de 1987. Na presente avaliação, os povoamentos possuem idade de 8,75 anos.

A área ocupada pelo experimento consta de seis blocos instalados de maneira contínua, em um total de 2,97 ha (incluindo a bordadura). Desta forma, tem-se:

Área de um bloco: $4.320 \mathrm{~m}^{2}$;

Área dos seis blocos: $25.920 \mathrm{~m}^{2}$;

Área dos seis blocos mais a bordadura: $29.692 \mathrm{~m}^{2}$.

O bloco refere-se à condição de produtividade do local. O bloco 6 corresponde ao maior valor médio de índice de sítio e o bloco 1 é a área com menor valor médio de índice de sítio.

O local de implantação do experimento possuía reflorestamento com Pinus taeda com 16 anos de idade. No final de 1986, executou-se um corte raso no projeto. A prática de limpeza do material resultante da exploração foi uma queima controlada, ficando a área segundo os critérios de reparo de terreno da empresa, apto a receber nova implantação.

A ferramenta principal utilizada para o plantio das mudas foi o enxadão, o qual propiciou a abertura de covas com dimensões médias de 20 em de largura por 15-20 em de profundidade em formato de funil. Não foi efetuado qualquer tipo de adubação.

Conforme a necessidade e dentro dos padrões adotados pela empresa, foram executadas limpezas no experimento.

Foram inicialmente mensuradas somente as alturas das árvores úteis das parcelas. Após as árvores atingirem altura superior a 1,3 m, as medições foram efetuadas obtendo-se os valores de CAP e altura total.

Os valores de CAP e altura foram mensurados nas datas fixadas na Tabela 1. O volume total e o sortimento foram estimados através de equações desenvolvidas para a região de Jaguariaíva. O experimento avalia cinco diferentes intensidades de espaçamentos, repetidos em seis blocos contínuos. Cada parcela possui bordadura dupla e são mensuradas somente as árvores constantes de sua área útil. Além disto, o teste possui uma bordadura dupla 
externa, procurando proporcionar o máximo de confiabilidade das inferências.

TABELA 1: Datas da coleta de dados do experimento.

\begin{tabular}{lc}
\hline Data de medição & Idade (anos) \\
\hline Maio/91 & 3,50 \\
Maio/92 & 4,50 \\
Outubro/93 & 5,92 \\
Outubro/94 & 6,92 \\
Julho/95 & 7,67 \\
Agosto/96 & 8,75 \\
\hline
\end{tabular}

A Tabela 2 apresenta os tratamentos do Delineamento em Blocos ao Acaso.

TABELA 2: Tratamentos analisados no experimento.

\begin{tabular}{ccc|c|c|c}
\hline Tratamento & Espaçamento $(\mathrm{m})$ & Árvores/ha & \multicolumn{2}{|c}{ Área da Parcela $\left(\mathrm{m}^{2}\right)$} & \multirow{2}{*}{ Plantas úteis } \\
\cline { 4 - 5 } & & & total & útil & \\
\hline 1 & $2,5 \times 1,2$ & 3333 & 630 & 234 & 78 \\
2 & $2,5 \times 2,0$ & 2000 & 630 & 210 & 42 \\
3 & $2,5 \times 2,8$ & 1428 & 810 & 315 & 45 \\
4 & $2,5 \times 3,6$ & 1111 & 990 & 378 & 42 \\
5 & $2,5 \times 4,4$ & 909 & 1260 & 440 & 40 \\
\hline
\end{tabular}

\section{Equações utilizadas nas estimativas de volume total e sortimento}

As estimativas de volume total e volume por sortimento obtidas até a idade atual dos povoamentos foram feitas através de equações ajustadas para povoamentos localizados na região de Jaguariaíva. Os ajustes das equações de volume e de forma consideraram uma série de dados de árvores distribuídas em diversas condições de idade, sítio e densidade. Essas funções têm sido utilizadas há vários anos na empresa onde o experimento foi instalado, e ajustadas para situações específicas a nível de espécie e local. Comparações com ajustes fornecidos por uma série de outras funções têm indicado a eficiência dos modelos aqui apresentados.

Para as idades subseqüentes, foram feitas simulações através do programa SISPINUS. Neste caso, os resultados da distribuição diamétrica prognosticados (valor central de cada classe de DAP, altura média correspondente e número de árvores por hectare estimado para cada classe) foram utilizados para estimar o "volume total por hectare" e o "volume por sortimento por hectare" na idade desejada.

A mesma função de volume utilizada até a idade atual dos 
povoamentos foi usada para estimar o volume total individual nas idades futuras (para o valor central de cada classe de DAP fornecido pelo SISPINUS). Multiplicando-se este volume pelo número de árvores de cada classe (prognosticado pelo SISPINUS), obteve-se o volume total por hectare por classe de DAP.

A soma dos volumes por hectare em todas as classes forneceu a variável necessária para o presente estudo.

O procedimento para o cálculo dos volumes por sortimento é o mesmo. As mesmas equações de forma utilizadas até a idade atual foram também usadas para as estimativas nas idades subseqüentes.

As equações de volume e de forma ajustadas para a região de Jaguariaíva são apresentadas na sequência.

1 - Equação para estimativa de volume individual:

$\mathrm{v}=\exp \left[\mathrm{b}_{\mathrm{o}}+\mathrm{b}_{1} \cdot \ln (\mathrm{DAP})+\mathrm{b}_{2} \cdot \ln (\mathrm{h})\right]$,

onde: $\mathrm{v}=$ volume total individual $\left(\mathrm{m}^{3}\right) ;{ }^{*} \mathrm{DAP}=$ diâmetro à altura do peito (em); $*$ h $=$ altura total $(\mathrm{m}) ; \mathrm{b}_{\mathrm{o}}, \mathrm{b}_{1}$ e $\mathrm{b}_{2}=$ coeficientes ajustados para povoamentos na área de estudo.

2 - Equação para estimativa da altura por sortimento:

$h_{c}=h-\left(f_{o} \cdot d_{\min }{ }^{f 1} \cdot h^{f 2}\right) / D A P^{f 3}$,

onde: $\quad h_{c}=$ altura comercial (m) para o sortimento desejado; $* h=$ altura total (m); $d_{\min }=$ diâmetro mínimo de uso (em) do sortimento desejado; $* D A P=$ diâmetro à altura do peito $(\mathrm{em}) ; f_{o^{\prime}}, f_{1}, f_{2}$ e $f_{3}=$ coeficientes ajustados para povoamentos na área de estudo.

3 - Equação para estimativa do volume por sortimento (individual):

$\mathrm{v}_{\mathrm{c}}=\mathrm{v} \cdot\left\{\left(1-\mathrm{p}_{\mathrm{O}}\right)\left[\left(\mathrm{h}-\mathrm{h}_{\mathrm{c}}\right)^{\mathrm{p} 1} /\left(\mathrm{h}^{\mathrm{p}}\right)\right]\right\}$

onde: $v_{c}=$ volume comercial $\left(\mathrm{m}^{3}\right)$ para o sortimento desejado; $* h=$ altura total $(\mathrm{m}) ; h_{s}=$ altura comercial $(\mathrm{m})$ para o sortimento desejado; $\mathrm{p}_{\mathrm{o}}$, $\mathrm{p}_{1}$ e $\mathrm{p}_{2}=$ coeficientes ajustados para povoamentos na área de estudo. * Valores mensurados até a idade atual e prognosticados pelo SISPINUS nas idades subseqüentes. No segundo caso, referem-se aos centros das classes de DAP.

\section{Análise da produção na idade de 8,75 anos}

Para a avaliação do experimento na idade de 8,75 anos, foi realizada 
a análise de variância para "volume total por hectare" e "volume para serraria por hectare". Para estes dois casos, aplicou- se o teste de comparação de médias dos tratamentos e dos blocos (Tukey ao nível de significância de 5\%).

\section{Análise da produção em idades futuras}

As situações definidas para as simulações foram as seguintes:

Entrada de dados (na idade de 8,75 anos)

Número de árvores por hectare e área basal de cada tratamento no bloco 1, e índice de sítio médio do bloco 1;

Número de árvores por hectare e área basal de cada tratamento no bloco 6, e índice de sítio médio do bloco 6;

Média do número de árvores por hectare e da área basal de cada tratamento em todos os blocos, e índice de sítio médio de todos os blocos. Os dados necessários para cada simulação são apresentados na Tabela 3.

TABELA 3: Dados iniciais utilizados para as simulações no programa SISPINUS.

\begin{tabular}{c|c|c|c|c|c|c}
\hline \multirow{2}{*}{ Tratamento } & \multicolumn{2}{|c|}{$\begin{array}{c}\text { Médias: } \\
\text { Todos os blocos } \\
\text { IS médio }=18,1 \mathrm{~m}\end{array}$} & \multicolumn{2}{c|}{$\begin{array}{c}\text { Bloco } 1 \\
\text { IS médio }=16,6 \mathrm{~m}\end{array}$} & \multicolumn{2}{c}{$\begin{array}{c}\text { Bloco 6 } \\
\text { IS médio }=19,6 \mathrm{~m}\end{array}$} \\
\cline { 2 - 7 } & $\begin{array}{c}\mathrm{G} \\
\left(\mathrm{m}^{2} / \mathrm{ha}\right)\end{array}$ & $\mathrm{N} / \mathrm{ha}$ & $\begin{array}{c}\mathrm{G} \\
\left(\mathrm{m}^{2} / \mathrm{ha}\right)\end{array}$ & $\mathrm{N} / \mathrm{ha}$ & $\mathrm{G}\left(\mathrm{m}^{2} / \mathrm{ha}\right)$ & $\mathrm{N} / \mathrm{ha}$ \\
\hline 1 & 48,4 & 3291 & 44,8 & 3333 & 50,0 & 3291 \\
2 & 43,3 & 2047 & 38,2 & 2000 & 49,2 & 2190 \\
3 & 37,3 & 1423 & 34,0 & 1492 & 36,9 & 1133 \\
4 & 33,7 & 1146 & 31,0 & 1111 & 38,0 & 1217 \\
5 & 30,4 & 932 & 28,8 & 909 & 31,2 & 955 \\
\hline
\end{tabular}

Esses dados são referentes à idade de 8,75 anos (= 9 anos no SISPINUS). IS $=$ Índice de Sítio (altura dominante na idade de referência de 15 anos).

Variáveis avaliadas

Como o regime de manejo adotado em povoamentos de Pinus taeda, na produção de madeira para celulose e papel, normalmente prescreve um corte raso aos 15 anos, utilizou-se esta idade para a avaliação das seguintes variáveis simuladas:

Volume total por hectare; 
Volume por sortimento por hectare.

Desta forma, as simulações foram feitas para possibilitar a avaliação da produção em três situações distintas de sítio: 1) bloco 1, que apresenta o menor valor médio de índice de sítio; 2 ) bloco 6 , onde é encontrado o maior valor médio de índice de sítio; e, 3) índice de sítio médio obtido de todos os blocos que compõem o experimento.

\section{RESULTADOS}

Os valores de produção em volume total e sortimento, estimados até a idade atual do experimento e simulados nas idades subseqüentes, são apresentados em anexo.

\section{Volume total por hectare aos 8,75 anos}

O Teste de Bartlett aplicado para o volume total indicou que as variâncias são homogêneas ao nível de significância de 5\%, tanto para os tratamentos corno para os blocos.

Foi utilizado o Delineamento em Blocos Casualizados (D.B.C.) para verificar diferenças entre os blocos e entre os tratamentos. Os resultados da análise de variância para volume total são apresentados na Tabela 4.

$\mathrm{O}$ teste $\mathrm{F}$ indicou que existem diferenças entre os tratamentos para o nível de significância de $1 \%$, o mesmo ocorrendo para os blocos. $\mathrm{O}$ valor de F é consideravelmente maior para os tratamentos, indicando que o efeito dos mesmos sobre o volume total é mais significativo que o efeito dos blocos. OsresultadosdotestedeTukeyparacomparaçãodasmédiasdostratamentosestão expressos na Tabela 5 .

O teste de Tukey realizado para a comparação de médias dos blocos forneceu os resultados apresentados na Tabela 6 . 
TABELA 4: Quadro da ANOVA para volume total por hectare, na idade de 8,75 anos.

\begin{tabular}{l|cc|c|c}
\hline \multicolumn{1}{c|}{ F.V. } & G.L. & S.Q. & Q.M. & F \\
\hline Tratamento & 4 & 29155,16 & 7288,79 & $36,39 * *$ \\
Bloco & 5 & 13054,28 & 2610,85 & $13,03 * *$ \\
Erro & 20 & 4005,94 & 200,29 & -- \\
Total & 29 & 46215,38 & -- & - \\
\hline
\end{tabular}

Em que: Tratamentos $=$ densidades iniciais de plantio; $\mathrm{SQ}=$ soma de quadrados; Blocos $=$ classes de sítio; $\mathrm{QM}=$ quadrados médios; $\mathrm{GL}=$ graus de liberdade; $\mathrm{F}=$ valor F calculado.

TABELA 5: Teste de Tukey para comparação de médias dos tratamentos ao nível de significância de $5 \%$, para o volume total por hectare, na idade de 8,75 anos.

\begin{tabular}{c|c|c|c|c}
\hline Tratamento & $\begin{array}{c}\text { Densidade inicial } \\
(\mathrm{N} / \mathrm{ha})\end{array}$ & $\begin{array}{c}\text { Volume médio } \\
\left(\mathrm{m}^{3} / \mathrm{ha}\right)\end{array}$ & $\begin{array}{c}\text { Média do IMA } \\
\left(\mathrm{m}^{3} / \text { ha/ano }\right)\end{array}$ & Comparações \\
\hline 1 & 3333 & 254,0 & 29,030 & $\mathrm{~A}$ \\
2 & 2000 & 241,2 & 27,568 & $\mathrm{~A}$ \\
3 & 1428 & 205,5 & 23,490 & $\mathrm{~B}$ \\
4 & 1111 & 185,8 & 21,228 & $\mathrm{~B}$ C \\
5 & 909 & 173,1 & 19,789 & $\mathrm{C}$ \\
\hline
\end{tabular}

Obs: os tratamentos com mesmas letras não diferem estatisticamente entre si.

TABELA 6: Teste de Tukey para comparação de médias dos blocos, ao nível de significância de $5 \%$, para o volume total por hectare, na idade de 8,75 anos.

\begin{tabular}{c|c|c|c}
\hline Bloco & $\begin{array}{c}\text { Volume médio } \\
\left(\mathrm{m}^{3} / \mathrm{ha}\right)\end{array}$ & $\begin{array}{c}\text { Média do IMA } \\
\left(\mathrm{m}^{3} / \text { ha/ano }\right)\end{array}$ & Comparações \\
\hline 5 & 238,1 & 27,065 & A \\
6 & 236,8 & 27,206 & A B \\
4 & 213,6 & 24,414 & A B C \\
3 & 209,8 & 23,975 & B C D \\
2 & 190,1 & 21,728 & C D \\
1 & 183,2 & 20,937 & D \\
\hline
\end{tabular}

Em que: os blocos com mesmas letras não diferem estatisticamente entre si. 


\section{Volume para serraria aos 8,75 anos}

Para esta variável, o teste de Bartlett indicou que as variâncias são homogêneas ao nível de significância de 5\%, para tratamentos e blocos.

A Análise de Variância para volume de serraria, na idade de 8,75 anos, é apresentada na Tabela 7.

TABELA 7: Quadro da ANOVA para volume de serraria por hectare, na idade de 8,75 anos.

\begin{tabular}{lccccc}
\hline \multicolumn{1}{c}{ F.V. } & G.L. & S.Q. & Q.M. & F \\
\hline Tratamento & 4 & 10712,16 & 2678,039 & $37,89^{* *}$ \\
Bloco & 5 & 2630,99 & 526,198 & $7,45^{* *}$ \\
Erro & 20 & 1413,50 & 70,675 & -- \\
Total & 29 & 14756,65 & - & - \\
\hline
\end{tabular}

Em que: Tratamentos $=$ densidades iniciais de plantio; $\mathrm{SQ}=$ soma de quadrados; Blocos = classes de sítio; $\mathrm{QM}=$ quadrados médios; $\mathrm{GL}=$ graus de liberdade; $\mathrm{F}=$ valor F calculado.

$\mathrm{O}$ teste $\mathrm{F}$ indicou diferenças significativas entre tratamentos e entre blocos, sendo que o valor de $\mathrm{F}$ foi mais significativo para os tratamentos.

O teste de comparação de médias dos tratamentos, para o volume de serraria por hectare, apresentou os resultados expressos na Tabela 8; e o teste de comparação de médias dos blocos forneceu os resultados apresentados na Tabela 9.

TABELA 8: Teste de Tukey para comparação de médias dos tratamentos ao nível de significância de $5 \%$, para o volume de serraria por hectare, na idade de 8,75 anos.

\begin{tabular}{c|c|c|c}
\hline Tratamento & $\begin{array}{c}\text { Densidade inicial } \\
(\mathrm{N} / \mathrm{ha})\end{array}$ & $\begin{array}{c}\text { "Vserraria" médio } \\
\left(\mathrm{m}^{3} / \mathrm{ha}\right)\end{array}$ & Comparaçōes \\
\hline 5 & 909 & 56,10 & $\mathrm{~A}$ \\
4 & 1111 & 45,93 & $\mathrm{~A} \mathrm{~B}$ \\
3 & 1428 & 34,40 & $\mathrm{~B} \mathrm{C}$ \\
2 & 2000 & 20,19 & $\mathrm{C}$ \\
1 & 3333 & 2,583 & $\mathrm{D}$ \\
\hline
\end{tabular}

Em que: os tratamentos com mesmas letras não diferem estatisticamente entre si. 
TABELA 9: Teste de Tukey para comparação de médias dos blocos, ao nível de significância de $5 \%$, para o volume de serraria por hectare, na idade de 8,75 anos.

\begin{tabular}{c|cc}
\hline Bloco & $\begin{array}{c}\text { "Vserraria" médio } \\
\left(\mathrm{m}^{3} / \text { ha }\right)\end{array}$ & Comparações \\
\hline 6 & 44,96 & A \\
5 & 40,38 & A \\
4 & 33,72 & A B \\
3 & 31,86 & A B \\
2 & 20,45 & B \\
1 & 19,68 & B \\
\hline
\end{tabular}

Em que: os blocos com mesmas letras não diferem estatisticamente entre si.

\section{Prognose da produção em volume total por hectare}

A produção em volume total por hectare em cada condição de sítio é apresentada nas Figuras de IA a IC. Os gráficos mostram a produção estimada até a idade atual do experimento (8,75 anos), e resultados de simulações com o programa SISPINUS para as idades subseqüentes.

Como os valores de diâmetro e altura foram obtidos de forma diferenciada nas simulações (não medidos diretamente no campo), pequenas discrepâncias foram percebidas, já que as curvas de produção apresentaram uma mudança de inclinação quando se passa da idade de 8,75 anos para a idade de 10 anos. Este fato, no entanto, não afeta o objetivo principal das simulações, que é proporcionar uma avaliação comparativa dos tratamentos de espaçamento em condições distintas de sítio nas idades futuras. 


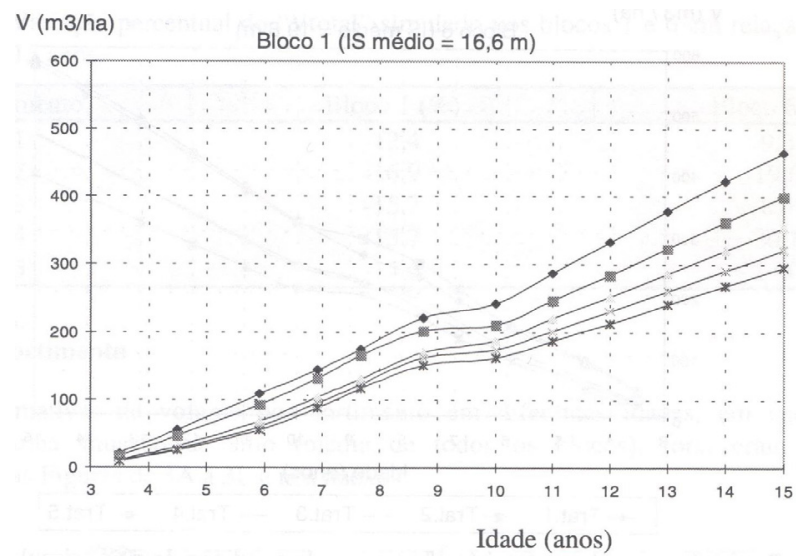

FIGURA 1A: Produção em volume total $\left(\mathrm{m}^{3} / \mathrm{ha}\right)$ em cada condição de sítio, simulado a partir de 11 anos (Legenda explicativa encontra-se na Figura 1C, na sequência).

$\mathrm{V}(\mathrm{m} 3 /$ ha $)$ Média de todos os blocos $(18$ médio $=18,1 \mathrm{~m})$

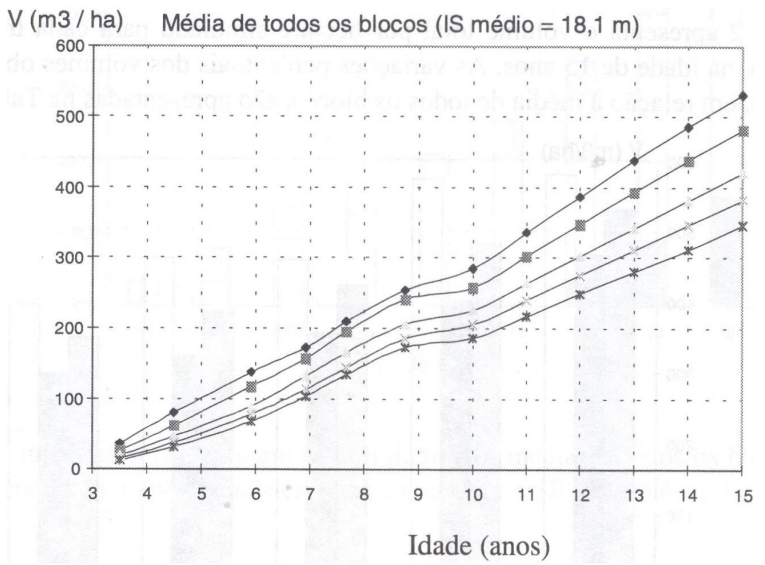

FIGURA 1B: Produção em volume total ( $\left.\mathrm{m}^{3} / \mathrm{ha}\right)$ em cada condição de sítio, simulado a partir de 11anos (Legenda explicativa encontra-se na Figura $1 \mathrm{C}$, na sequência). 


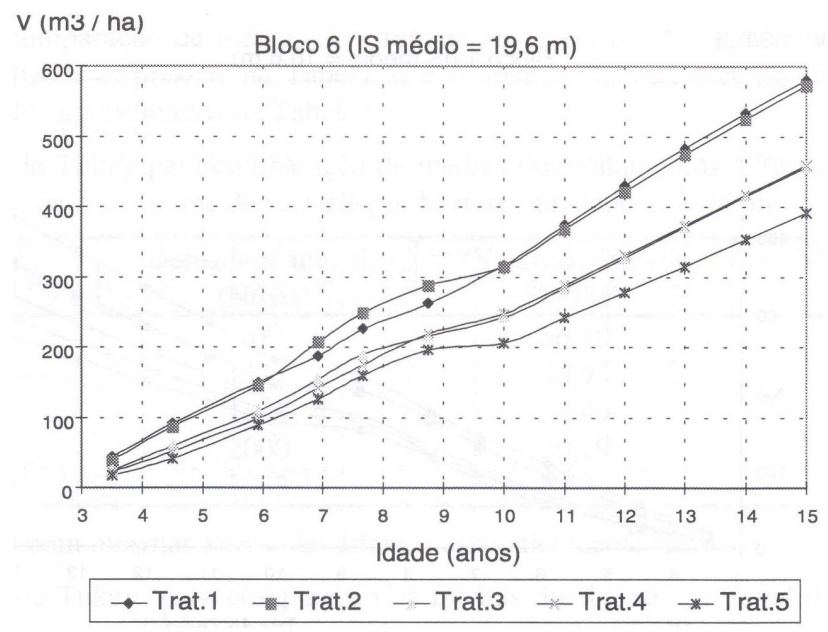

FIGURA 1C: Produção em volume total ( $\left.\mathrm{m}^{3} / \mathrm{ha}\right)$ em cada condição de sítio, simulado a partir de 11 anos.

Efeito das condições extremas de sítio sobre o volume total, na idade de 15 anos

A Figura 2 apresenta o volume total por hectare simulado para cada tratamento, em cada condição de sítio, na idade de 15 anos. As variações percentuais dos volumes obtidos em condições extremas de sítio, em relação à média de todos os blocos, são apresentadas na Tabela 10 . 


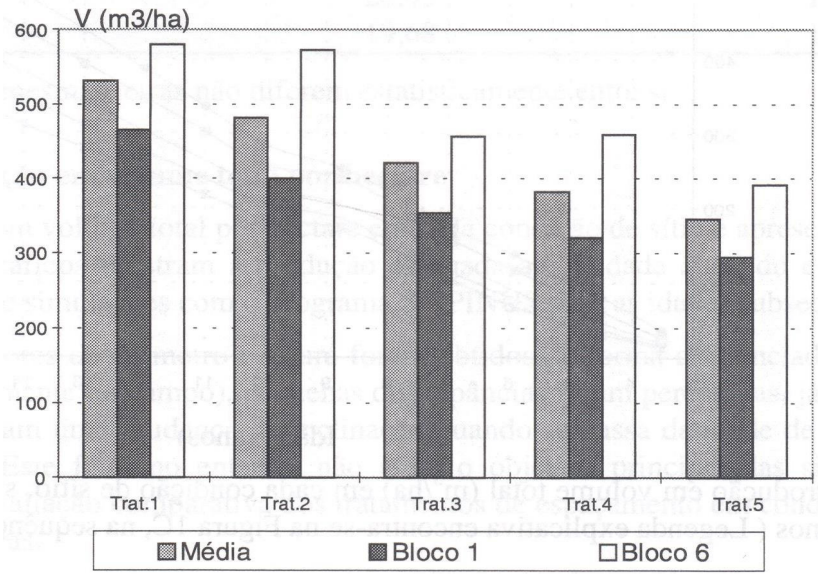

FIGURA 2: Volume total por hectare simulado para a idade de 15 anos.

TABELA 10: Variação percentual do "Vtotal" simulado nos blocos 1 e 6 em relação à média, aos 15 anos.

\begin{tabular}{c|c|c}
\hline Tratamento & Bloco 1 $(\%)$ & Bloco 6 (\%) \\
\hline 1 & $-12,4$ & 9,3 \\
2 & $-16,9$ & 19,0 \\
3 & $-15,7$ & 8,4 \\
4 & $-15,7$ & 20,1 \\
5 & $-14,6$ & 13,0 \\
\hline
\end{tabular}

\section{Evolução do sortimento}

As estimativas de volume por sortimento em diferentes idades, em cada tratamento, considerando uma situação de sítio (média de todos os blocos), forneceram os resultados apresentados nas Figuras de $3 \mathrm{~A}$ a $3 \mathrm{E}$ e nos anexos. 
Gomes, F. S.; Maestre, R.; Sanquetta, C. R.

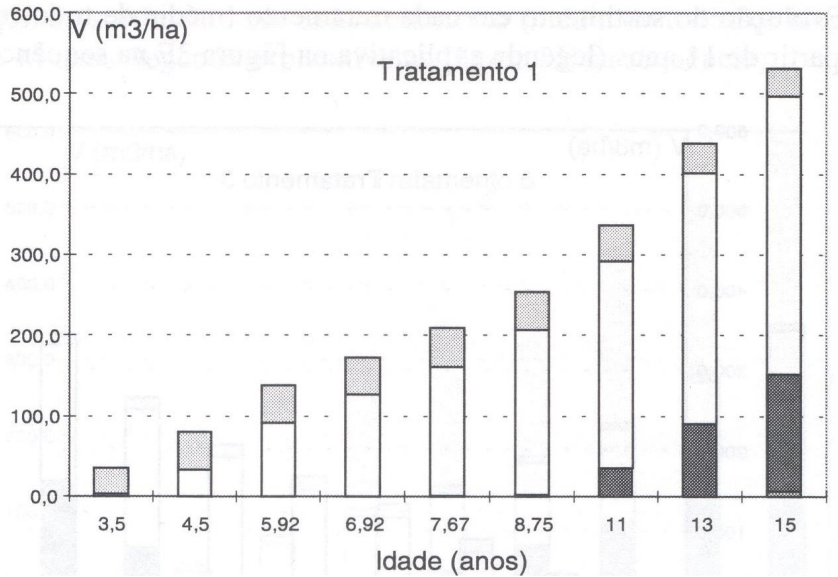

FIGURA 3A: Evolução do sortimento em cada tratamento (média de todos os blocos) simulado a partir de 11 anos (legenda explicativa na Figura 3E na sequência).

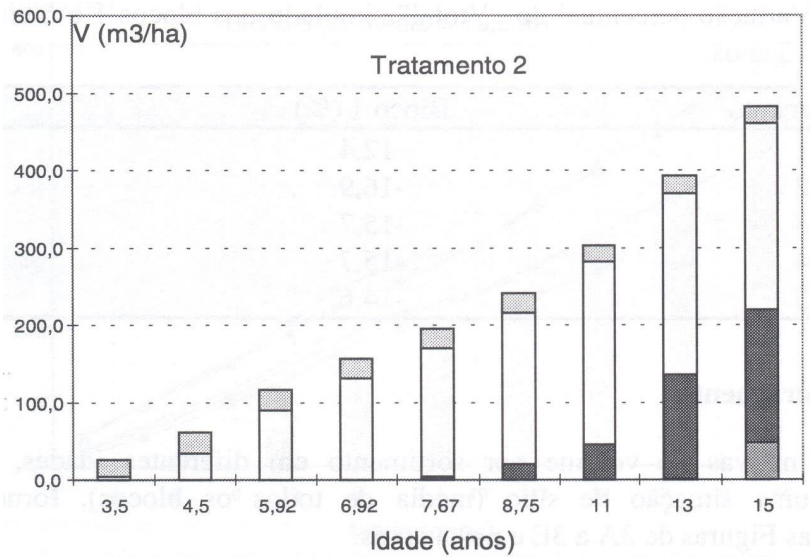

FIGURA 3B: Evolução do sortimento em cada tratamento (média de todos os blocos) - simulado a partir de 11 anos (legenda explicativa na Figura 3E na sequência). 


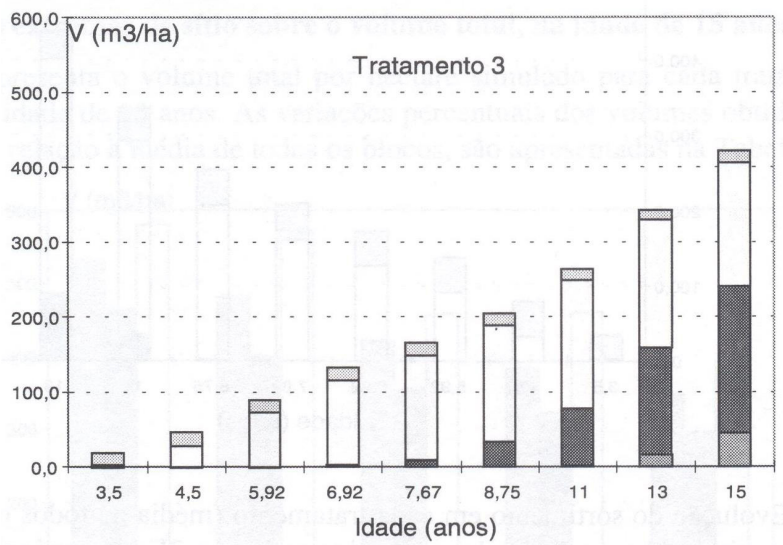

FIGURA 3C: Evolução do sortimento em cada tratamento (média de todos os blocos) - simulado a partir de 11 anos (legenda explicativa na Figura 3E na sequência).

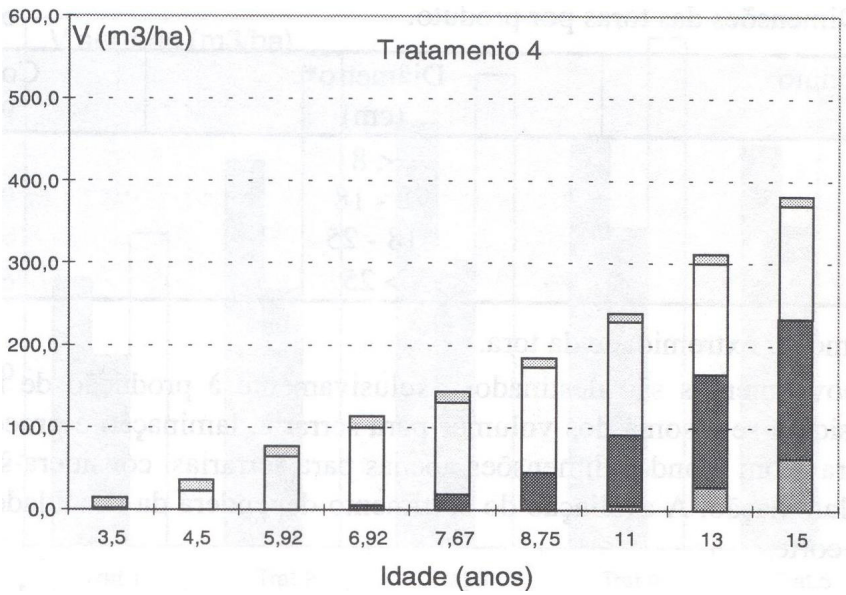

FIGURA 3D: Evolução do sortimento em cada tratamento (média de todos os blocos) - simulado a partir de 11 anos (legenda explicativa na Figura 3E na sequência). 


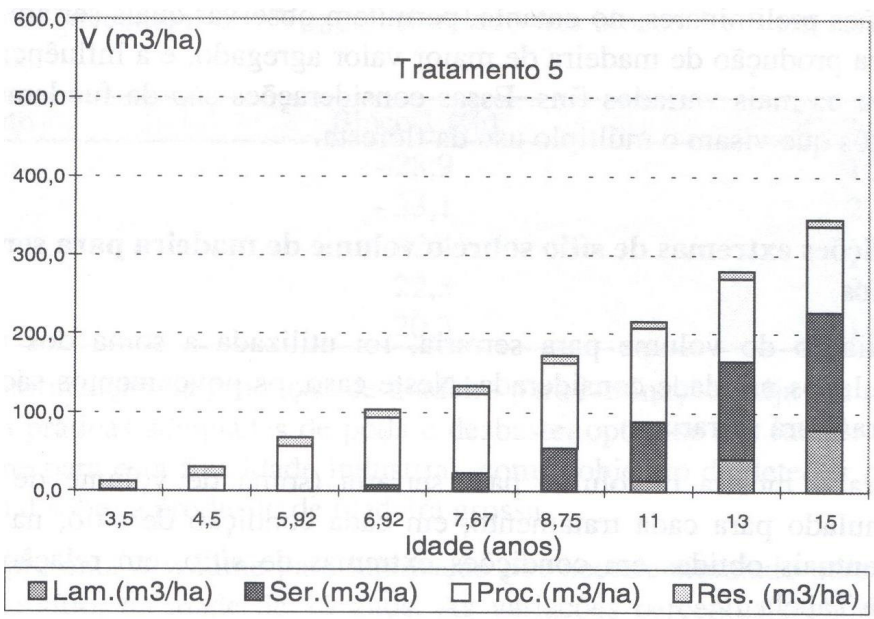

FIGURA 3E: Evolução do sortimento em cada tratamento (média de todos os blocos) - simulado a partir de 11 anos.

As dimensões das toras para cada finalidade industrial são apresentadas na Tabela 11 .

TABELA 11: Dimensões das toras por produto.

\begin{tabular}{l|c|c}
\hline & $\begin{array}{c}\text { Diâmetro* } \\
(\mathrm{cm})\end{array}$ & Comprimento (m) \\
\hline Resíduo & $<8$ & - \\
Processo & $8-18$ & 1,20 \\
Serraria & $18-25$ & 3,10 \\
Laminação & $>25$ & 2,70 \\
\hline
\end{tabular}

Em que: *Diâmetro na menor extremidade da tora.

Se os povoamentos são destinados exclusivamente à produção de madeira para processo (celulose), considera-se a soma dos volumes para serraria, laminação e processo. Se o objetivo é a produção de toras com grandes dimensões apenas para serrarias, considera-se a soma dos volumes para serraria e laminação. A avaliação do sortimento dependerá da finalidade industrial da madeira proveniente do corte.

Deve-se ressaltar que o estudo do experimento aqui apresentado contempla de forma principal a produção de madeira exclusivamente para 
celulose, uma vez que desbastes não foram efetuados nas parcelas. Simulações de desbastes poderiam ser consideradas, mas aumentariam substancialmente o número de alternativas de regimes de manejo.

Avaliações preliminares, no entanto, permitem observar quais seriam os espaçamentos mais apropriados para produção de madeira de maior valor agregado, e a influência do sítio na produção de madeira para os mais variados fins. Essas considerações são de fundamental importância para empreendimentos que visam o múltiplo uso da floresta.

\section{Efeito de condições extremas de sítio sobre o volume de madeira para serraria e laminação, na idade de 15 anos}

$\mathrm{Na}$ avaliação do volume para serraria, foi utilizada a soma dos volumes de serraria e laminação simulados na idade considerada. Neste caso, os povoamentos são destinados somente à produção de toras para serraria.

A Figura 4 mostra o volume para serraria (soma de volume de serraria e volume de laminação) simulado para cada tratamento, em cada condição de sítio, na idade de 15 anos. As variações percentuais obtidas em condições extremas de sítio, em relação à média de todos os blocos, estão na Tabela 12.

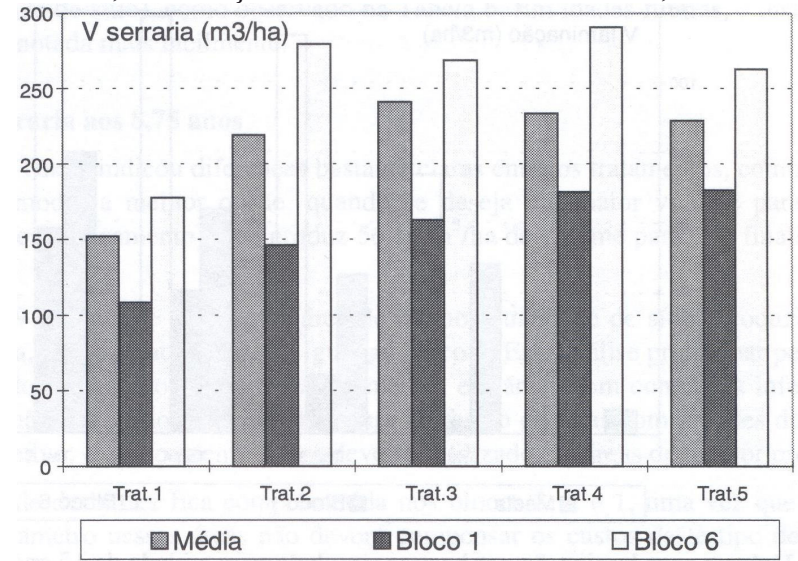

FIGURA 4: Volume para serraria (ser.+ lam) por hectare, simulado para a idade de 15 anos. 
TABELA 12: Variação percentual do "V $\mathrm{V}_{\text {serraria }}$ simulado nos blocos 1 e 6 em relação à média, aos 15 anos.

\begin{tabular}{c|cc}
\hline Tratamento & Bloco 1 (\%) & Bloco 6 (\%) \\
\hline 1 & $-28,9$ & 16,8 \\
2 & $-33,1$ & 27,5 \\
3 & $-32,4$ & 11,6 \\
4 & $-22,3$ & 24,5 \\
5 & $-20,3$ & 14,8 \\
\hline
\end{tabular}

Embora a maximização da produção de madeira para laminação exija um regime de manejo mais complexo, com práticas adequadas de poda e desbaste, optou-se por incluir os resultados das simulações de volume para esta finalidade industrial, com o objetivo de detectar o efeito do sítio e do espaçamento inicial sobre a produção de madeira grossa.

A Figura 5 apresenta o volume para laminação por hectare simulado para cada tratamento, em cada condição de sítio, na idade de 15 anos. As variações percentuais obtidas em condições extremas de sítio, em relação à condição média, são apresentadas na Tabela 13.

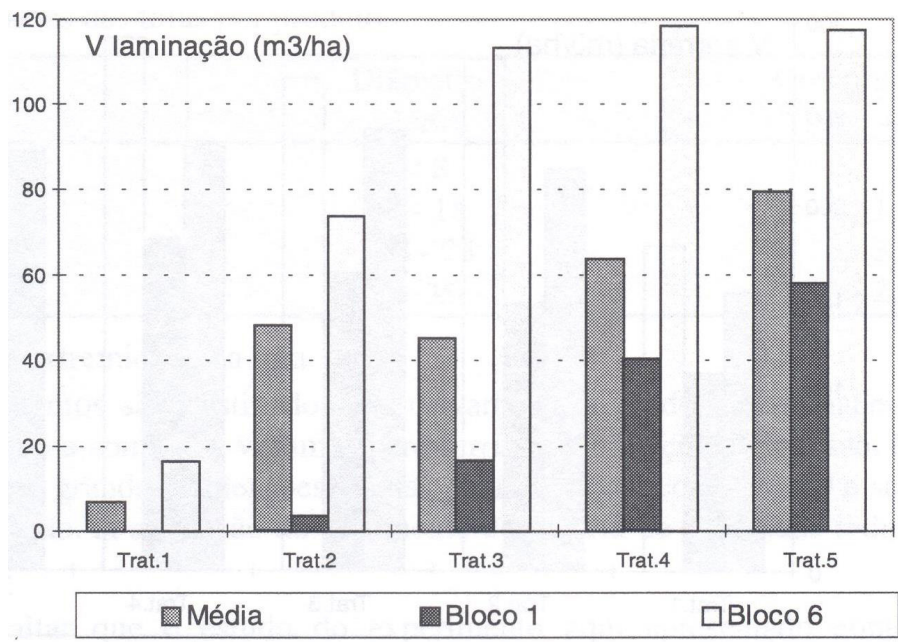

FIGURA 5: Volume para laminação por hectare simulado para a idade de 15 anos. 
TABELA 13: Variação percentual do "V $\mathrm{V}_{\text {laminacăa }}$ simulado nos blocos 1 e 6 em relação à média, aos 15 anos.

\begin{tabular}{c|c|c}
\hline Tratamento & Bloco 1 (\%) & Bloco 6 (\%) \\
\hline 1 & $-100,0$ & 140,3 \\
2 & $-92,4$ & 53,4 \\
3 & $-63,3$ & 150,2 \\
4 & $-36,6$ & 85,8 \\
5 & $-27,1$ & 47,6 \\
\hline
\end{tabular}

\section{DISCUSSÃO}

\section{Volume total aos 8,75 anos}

O teste de Tukey indicou que os tratamentos 1 e 2 são estatisticamente iguais, fornecendo volumes de 254,0 e $241,2 \mathrm{~m}^{3} \mathrm{lha}$, como observado na Tabela 5. O tratamento 3 é intermediário, com volume de $205,5 \mathrm{~m}^{3} / \mathrm{ha}$; e os tratamentos 4 e 5 fornecem os menores valores, sendo estatisticamente iguais (185,8 e $\left.173,1 \mathrm{~m}^{3} \mathrm{lha}\right)$.

O tratamento 2 toma-se então o mais viável para produção em volume total, pois requer uma menor densidade de plantio em relação ao tratamento 1. Esta conclusão, no entanto, é válida para a idade de 8,75 anos, tomando necessária uma avaliação da produção em idades futuras.

O teste de comparação de médias, na idade de 8,75 anos, indicou que existem diferenças significativas entre os blocos, demonstrando que a consideração dos mesmos no experimento é necessária. O bloco 5 produziu um volume total maior, sendo estatisticamente igual ao bloco 6 (área de melhor qualidade de sítio), como observado na Tabela 6. Em idades futuras, a distinção entre os blocos deverá ser notada mais facilmente.

\section{Volume para serraria aos 8,75 anos}

$\mathrm{O}$ teste de Tukey indicou diferenças bastante claras entre os tratamentos, como observado na Tabela 8. Deste modo, a melhor opção, quando se deseja um maior volume para serraria, é o tratamento 5 (maior espaçamento), que produz $56,10 \mathrm{~m}^{3} /$ ha de volume para esta finalidade aos 8,75 anos.

A Tabela 9 indica que o bloco 6 (área de melhor qualidade de 
sítio) produziu um volume maior para serraria, sendo estatisticamente igual ao bloco 5. Esta análise preliminar permite concluir que a utilização do tratamento 5 (maior espaçamento) em áreas com condições inferiores de sítio (blocos 3, 2 e 1) não é a opção mais indicada para produção de toras com grandes dimensões. Para atender a este objetivo, o espaçamento maior deve ser utilizado em áreas de melhor produtividade.

A prática de desbastes fica comprometida nos blocos 3,2 e 1, uma vez que a resposta do incremento em diâmetro nessas áreas não deverá compensar os custos deste tipo de operação. Os blocos 5 e 6 são mais adequados para produzir madeira de maior valor agregado.

\section{Volume total simulado aos 15 anos}

O volume total simulado para a idade de 15 anos apresenta significativa influência das condições extremas de sítio, como mostram a Figura 2 e a Tabela 10. A variação percentual do volume obtido no "bloco 1" em relação ao encontrado na "média de todos os blocos" chega a 16,9\% no tratamento 2, por exemplo. Para este mesmo tratamento, existe uma diferença de 19,0\% entre o "bloco 6" e a "média de todos os blocos".

Nas áreas de maior produtividade (bloco 6), os tratamentos 1 e 2 apresentaram curvas de produção volumétrica bastante próximas, como pode ser visto na Figura 1. Desta forma, a utilização de áreas com melhores condições de sítio, visando a maximização da produção em volume total, possibilita o uso de uma densidade inicial de plantio igual à utilizada no tratamento 2 (2.000 árvores por hectare). Para terrenos com qualidade de sítio inferior, o tratamento 1 (3.333 árvores por hectare) é o mais adequado.

\section{Evolução do sortimento}

Esta análise considerou o volume para laminação, serraria, processo e residual, em função do tempo, como mostra a Figura 3. Os resultados são provenientes da média de todos os blocos.

A produção de volume para serraria nos tratamentos 4 e 5 (espaçamentos mais amplos) tem início na idade de 6,92 anos. No tratamento 2 , esta produção começa na idade de 8,75 anos, ou seja, aproximadamente dois anos mais tarde, e no tratamento 1 (maior densidade inicial), o volume para serraria é detectado somente nos resultados das simulações. 
Analisando-se os tratamentos em uma determinada idade, verifica-se que a utilização de espaçamentos mais amplos acarreta um aumento do volume disponível para fins mais nobres. Na idade de 8,75 anos, o volume para serraria aumenta quando o espaçamento é maior (Figura 3). Em idades futuras, no entanto, esta tendência não é verificada. A Figura 4 permite observar que, aos 15 anos, o efeito dos tratamentos 3,4 e 5 é praticamente o mesmo na produção de madeira para esta finalidade, o que se deve principalmente à não realização de desbastes. $\mathrm{O}$ volume total, em qualquer idade, diminui com o aumento do espaçamento.

\section{Volume por sortimento simulado aos 15 anos}

O volume para serraria disponível aos 15 anos de idade é consideravelmente influenciado pelas condições extremas de sítio, conforme observado na Figura 4 e na Tabela 12. No tratamento 2, as diferenças verificadas na produção em volume para esta finalidade são de $33,1 \%$ entre o bloco 1 e média, e $27,0 \%$ entre o bloco 6 e a média. Esta variação percentual é maior que a verificada para volume total.

As maiores diferenças percentuais existentes entre as condições extremas de sítio são detectadas na produção de madeira para laminação, conforme ilustrado na Figura 5 e Tabela 13. Para o tratamento 2, tomado como exemplo, esta diferença chega a $92,4 \%$ entre o bloco 1 e a média, e $53,4 \%$ entre o bloco 6 e a média. A influência das condições de sítio, deste modo, é mais evidente na produção de madeira de maior valor agregado.

\section{CONCLUSÕES}

A avaliação realizada na idade de 8,75 anos demonstrou que os blocos 5 e 6 (áreas com melhores condições de sítio) forneceram os melhores resultados em termos de volume total. A maior produção volumétrica, nesta idade, pode ser alcançada através do tratamento 2 (2000 árvores por hectare), nos blocos 5 e 6 . A escolha do tratamento 2 reduz os custos de implantação em relação ao tratamento 1 (3333 árvores por hectare). Os resultados das simulações para as idades subseqüentes indicaram o tratamento 2 como o mais apropriado na produção em volume total, para áreas com boas condições de declividade (bloco 6). Desta forma, o espaçamento inicial pode ser definido para cada condição de sítio. 
O volume total é consideravelmente afetado pelas condições de sítio. A produção sofre significativa influência do sítio, na idade de 8,75 anos. Os resultados das simulações para a idade de 15 anos, revelaram diferenças percentuais significativas entre os volumes obtidos em condições extremas de produtividade (blocos 1 e 6) em relação à produtividade média de todos os blocos.

No teste de comparação de médias realizado para volume de serraria na idade de 8,75 anos, verificou-se diferenças significativas entre os tratamentos. Nas simulações realizadas para a idade de 15 anos, no entanto, os tratamentos apresentaram-se próximos, indicando que a não realização de desbastes inibe o efeito do espaçamento inicial na produção de volume para serraria.

Na idade de 8,75 anos, já é percebido o efeito do sítio na produção de madeira para serraria. As simulações realizadas para a idade de 15 anos indicaram que na produção de madeira de maior valor agregado, o efeito do sítio é mais evidente. Povoamentos a serem manejados com regimes de poda e desbaste devem ser implantados preferencialmente em áreas com boas condições de produtividade.

Se o objetivo é produzir somente madeira para processo (celulose e papel) ou para energia, os espaçamentos mais densos são mais apropriados, no que se refere à maximização da produção física (volume total). A produção de madeira serrada ou laminada, por outro lado, toma-se mais viável em povoamentos com maior espaçamento inicial. Neste caso, o regime de desbastes deve estar intimamente associado à densidade de plantio.

O máximo aproveitamento do potencial produtivo do solo deve ser considerado na escolha da densidade de plantio. Sítios melhores serão melhor aproveitados na produção de madeira para fins mais nobres. A prática de desbastes terá melhores respostas, em termos de incremento diamétrico, em áreas mais produtivas. As condições de topografia do terreno devem ser corretamente analisadas, pois definirão aspectos de exploração e de utilização ou não de práticas de desbastes. A escolha da densidade inicial dependerá também de questões relacionadas aos custos de transporte e de exploração. A finalidade da madeira produzida, a distância ao mercado consumidor (serrarias, laminadoras ou fábrica de papel), aliadas à maior ou menor dificuldade de exploração (desbastes e corte final), devem definir práticas silviculturais e, conseqüentemente, a densidade inicial a ser adotada.

Cabe ainda ressaltar a importância de um estudo adicional de variáveis 
relacionadas à qualidade dos fustes nos povoamentos deste experimento. $\mathrm{O}$ percentual do número de árvores existente para cada classe de qualidade de fuste, e o percentual do número de árvores bifurcarias, quebradas e mortas, são variáveis analisadas em um estudo paralelo.

A avaliação econômica de diferentes regimes de manejo aplicados em situações distintas de sítio irá complementar o presente estudo. A análise de custos em diferentes condições de localização dos povoamentos florestais, propiciará a definição de um regime de manejo (espaçamento "inicial e idade de rotação) considerado ideal para cada classe de distância do mercado consumidor (fábrica de papel).

\section{AGRADECIMENTOS}

Os autores desejam agradecer à empresa Pisa Florestal por ter permitido gentilmente a utilização dos dados do experimento de espaçamento neste trabalho.

\section{REFERÊNCIAS BIBLIOGRAFIAS}

AHRENS, S. A concepção de regimes de manejo para plantações de Pinus spp, no Brasil. Curitiba, EMBRAPA-CNPFlorestas. 23p. (Circular Técnica, 10). 1985.

DANIEL, P.W., HELMS, D.E., BAKER, F.S. Principios de silvicultura. México: Ed Mc Graw Hill. 492 p. 1982.

DAVIS, K. P. Forest management. USA: Ed Me Graw Hill. 519 p. 1966. HUSCH, B.; MILLER, C. I. \& BEERS, T. W. Forest Mensuration. USA: John Wiley \& Sons. 402p. 1982.

OLIVEIRA, E. B. Um sistema computadorizado de prognose do crescimento e produção de Pinus taeda L., com critérios quantitativos para a avaliação técnica e econômica de regimes de manejo. Curitiba. 134 p. Tese de Doutorado, Universidade Federal do Paraná. 1995. 


\section{ANEXOS}

Volume total $\left(\mathrm{m}^{3} / \mathrm{ha}\right)$ - simulado a partir de 10 anos.

\begin{tabular}{|c|c|c|c|c|c|c|c|c|c|c|c|c|}
\hline \multicolumn{13}{|c|}{ Média de todos os blocos ( IS médio $=18,1 \mathrm{~m}$ ) } \\
\hline \multirow[t]{2}{*}{ Tratamento } & \multicolumn{12}{|c|}{ Idade (anos) } \\
\hline & 3,5 & 4,5 & 5,92 & 6,92 & 7,67 & 8,75 & 10,0 & 11,0 & 12,0 & 13,0 & 14,0 & 15,0 \\
\hline 1 & 36,2 & 80 & 137,8 & 172,8 & 209,3 & 254 & 284,9 & 336,5 & 387,1 & 438,7 & 486,4 & 531,4 \\
\hline 2 & 26 & 61,9 & 116,8 & 157,2 & 195,9 & 241,2 & 258 & 303 & 348,2 & 393,7 & 438,3 & 482,2 \\
\hline 3 & 18,9 & 46,7 & 89,4 & 132,5 & 166 & 205,5 & 225,9 & 264 & 302,8 & 342,3 & 381,7 & 421,5 \\
\hline 4 & 14,7 & 37,4 & 77,5 & 113,6 & 144,6 & 185,8 & 205,9 & 239,8 & 275,4 & 311,6 & 346,8 & 382,5 \\
\hline \multirow[t]{2}{*}{5} & 12 & 31,4 & 67,9 & 103,8 & 134,5 & 173,1 & 186,2 & 217,1 & 249 & 280,9 & 311,7 & 346,7 \\
\hline & \multicolumn{12}{|c|}{ Bloco 1 ( IS médio = 16,6m ) } \\
\hline \multirow[t]{2}{*}{ Tratamento } & \multicolumn{12}{|c|}{ Idade (anos) } \\
\hline & 3,5 & 4,5 & 5,92 & 6,92 & 7,67 & 8,75 & 10,0 & 11,0 & 12,0 & 13,0 & 14,0 & 15,0 \\
\hline 1 & 20,9 & 55,2 & 108,2 & 143,6 & 174,6 & 221,3 & 242,9 & 287,4 & 333,6 & 378,9 & 423,5 & 465,7 \\
\hline 2 & 16,5 & 46,4 & 92,4 & 131,9 & 165,1 & 202 & 210,7 & 247,5 & 284,9 & 324 & 362,7 & 400,7 \\
\hline 3 & 10,6 & 30,5 & 68,1 & 103,9 & 130,7 & 170,1 & 187,9 & 220,5 & 252,8 & 286,9 & 321 & 355,4 \\
\hline 4 & 8,9 & 26,7 & 60 & 94,8 & 122,2 & 162,1 & 173,8 & 201,8 & 231,8 & 260,7 & 291,1 & 322,4 \\
\hline \multirow[t]{2}{*}{5} & 8,9 & 25,4 & 54,9 & 87,8 & 116,2 & 150,5 & 161,7 & 187,2 & 213,5 & 241,4 & 268,4 & 296 \\
\hline & \multicolumn{12}{|c|}{ Bloco $6($ IS médio $=19,6 \mathrm{~m})$} \\
\hline \multirow[t]{2}{*}{ Tratamento } & \multicolumn{12}{|c|}{ Idade (anos) } \\
\hline & 3,5 & 4,5 & 5,92 & 6,92 & 7,67 & 8,75 & 10,0 & 11,0 & 12,0 & 13,0 & 14,0 & 15,0 \\
\hline 1 & 45,1 & 92,3 & 150,5 & 188,2 & 226,4 & 262,8 & 317,5 & 374,7 & 430,5 & 484,1 & 534,1 & 580,8 \\
\hline 2 & 40,2 & 87,5 & 146,7 & 207,4 & 249,7 & 288,4 & 315,7 & 368,7 & 421,6 & 474 & 525,3 & 573,6 \\
\hline 3 & 25,7 & 60,4 & 110,4 & 155,2 & 191,4 & 216,7 & 242,9 & 284,9 & 327,8 & 372 & 415,1 & 457,1 \\
\hline 4 & 22,9 & 52,7 & 99,4 & 142,9 & 176,1 & 219,5 & 248,5 & 288,9 & 331,2 & 374,2 & 417,6 & 459,3 \\
\hline 5 & 18 & 42,8 & 90 & 126,8 & 160,7 & 196,7 & 206,8 & 242,9 & 278,9 & 315,3 & 354,1 & 391,9 \\
\hline
\end{tabular}

Continuação.

\begin{tabular}{|c|c|c|c|c|c|c|c|c|c|c|c|c|}
\hline \multicolumn{13}{|c|}{ Tratamento 4} \\
\hline \multirow{2}{*}{$\begin{array}{l}\text { Idade } \\
\text { (anos) }\end{array}$} & \multicolumn{4}{|c|}{ Média de todos os blocos } & \multicolumn{4}{|c|}{ Bloco 1} & \multicolumn{4}{|c|}{ Bloco 6} \\
\hline & Lam. & Ser. & Proc. & Res. & Lam. & Ser. & Proc. & Res. & Lam. & Ser. & Proc. & Res. \\
\hline 3,5 & 0,0 & 0,0 & 2,6 & 12,1 & 0,0 & 0,0 & 0,0 & 14,7 & 0,0 & 0,0 & 4,6 & 10,1 \\
\hline 4,5 & 0,0 & 0,0 & 23,4 & 14,0 & 0,0 & 0,0 & 16,5 & 20,9 & 0,0 & 0,0 & 27,2 & 10,2 \\
\hline 5,92 & 0,0 & 0,0 & 64,5 & 13,0 & 0,0 & 0,0 & 59,4 & 18,1 & 0,0 & 0,0 & 67,1 & 10,4 \\
\hline 6,92 & 0,0 & 5,8 & 94,5 & 13,3 & 0,0 & 0,0 & 97,3 & 16,3 & 0,0 & 7,1 & 95,6 & 10,9 \\
\hline 7,67 & 0,0 & 19,5 & 111,6 & 13,5 & 0,0 & 10,2 & 118,7 & 15,7 & 0,0 & 27,0 & 105,2 & 12,4 \\
\hline 8,75 & 0,0 & 45,9 & 127,6 & 12,3 & 0,0 & 35,3 & 136,6 & 13,9 & 0,0 & 55,1 & 120,2 & 10,5 \\
\hline 11,0 & 5,8 & 86,4 & 137,4 & 10,3 & 1,4 & 72,2 & 155,0 & 11,2 & 11,9 & 104,4 & 113,5 & 10,1 \\
\hline 13,0 & 29,3 & 136,4 & 134,0 & 11,9 & 18,3 & 122,0 & 158,5 & 12,8 & 39,4 & 141,6 & 120,6 & 10,0 \\
\hline \multirow[t]{2}{*}{15,0} & 63,7 & 169,9 & 137,6 & 11,3 & 47,9 & 167,5 & 154,1 & 13,0 & 98,5 & 143,7 & 130,5 & 9,8 \\
\hline & \multicolumn{12}{|c|}{ Tratamento 5} \\
\hline \multirow{2}{*}{$\begin{array}{l}\text { Idade } \\
\text { (anos) }\end{array}$} & \multicolumn{4}{|c|}{ Média de todos os blocos } & \multicolumn{4}{|c|}{ Bloco 1} & \multicolumn{4}{|c|}{ Bloco 6} \\
\hline & Lam. & Ser. & Proc. & Res. & Lam. & Ser. & Proc. & Res. & Lam. & Ser. & Proc. & Res \\
\hline 3,5 & 0,0 & 0,0 & 1,7 & 10,3 & 0,0 & & 0,0 & 12,0 & 0,0 & 0,0 & 3,6 & 8,4 \\
\hline 4,5 & 0,0 & 0,0 & 19,7 & 11,7 & 0,0 & 0,0 & 16,3 & 15,1 & 0,0 & 0,0 & 22,7 & 8,7 \\
\hline 5,92 & 0,0 & 0,0 & 57,3 & 10,6 & 0,0 & 0,0 & 55,9 & 12,0 & 0,0 & 3,0 & 57,3 & 7,6 \\
\hline 6,92 & 0,0 & 7,1 & 86,1 & 10,6 & 0,0 & 0,0 & 92,0 & 11,8 & 0,0 & 17,3 & 77,7 & 8,8 \\
\hline 7,67 & 0,0 & 23,9 & 100,2 & 10,4 & 0,0 & 14,9 & 107,9 & 11,7 & 0,0 & 34,0 & 91,6 & 8,9 \\
\hline 8,75 & 0,0 & 55,0 & 107,9 & 10,3 & 0,0 & 49,0 & 113,2 & 10,9 & 0,0 & 63,6 & 99,9 & 9,6 \\
\hline 11,0 & 13,2 & 75,5 & 119,4 & 9,0 & 7,6 & 77,8 & 120,7 & 11,0 & 16,4 & 97,1 & 95,6 & 8,0 \\
\hline & 41,6 & 123,9 & 106,0 & 9,4 & 32,3 & 101,7 & 137,5 & 9,5 & 46,8 & 126,3 & 99,6 & 8,2 \\
\hline 15,0 & 79,6 & 149,4 & 108,8 & 8,8 & 68,0 & 145,8 & 123,0 & 9,8 & 103,9 & 128,7 & 106,4 & 7,7 \\
\hline
\end{tabular}

Ci. Fl., v. 7, n. 1, 1997 